\title{
Proteomic analysis of selective cytotoxic anticancer properties of flavonoids isolated from Citrus platymamma on A549 human lung cancer cells
}

\author{
ARULKUMAR NAGAPPAN ${ }^{1}$, VENU VENKATARAME GOWDA SARALAMMA ${ }^{2}$, GYEONG EUN HONG ${ }^{2}$, \\ HO JEONG LEE ${ }^{2}$, SUNG CHUL SHIN ${ }^{3}$, EUN HEE KIM ${ }^{4}$, WON SUP LEE ${ }^{1}$ and GON SUP KIM ${ }^{2}$ \\ ${ }^{1}$ Department of Internal Medicine, Institute of Health Sciences and Gyeongnam Regional Cancer Center, \\ Gyeongsang National University School of Medicine, Jinju, Gyeongnam 660-702; \\ ${ }^{2}$ Research Institute of Life Science and College of Veterinary Medicine (BK21 Plus Project); \\ ${ }^{3}$ Department of Chemistry, Research Institute of Life Science, Gyeongsang National University, Jinju, Gyeongnam 660-701; \\ ${ }^{4}$ Department of Nursing Science, International University of Korea, Jinju, Gyeongnam 660-759, Republic of Korea
}

Received July 9, 2015; Accepted June 29, 2016

DOI: $10.3892 / \mathrm{mmr} .2016 .5666$

\begin{abstract}
Citrus platymamma Hort. ex Tanaka (Byungkyul in Korean) has been used in Korean folk medicine for the treatment of inflammatory disorders and cancer. However, the molecular mechanism underlying the anticancer properties of flavonoids isolated from C. platymamma (FCP) remains to be elucidated. Therefore, the present study attempted to identify the key proteins, which may be important in the anticancer effects of FCP on A549 cells using a proteomic approach. FCP showed a potent cytotoxic effect on the A549 human lung cancer cells, however, it had no effect on WI-38 human fetal lung fibroblasts at the same concentrations. Furthermore, 15 differentially expressed protein spots (spot intensities $\geq 2$-fold change; $\mathrm{P}<0.05$ ) were obtained from comparative proteome analysis of two-dimensional gel electrophoresis maps of the control (untreated) and FCP-treated A549 cells. Finally, eight differentially expressed proteins, one of which was upregulated and seven of which were downregulated, were successfully identified using matrix-assisted laser desorption/ionization time-of-flight/time-of-flight tandem mass spectrometry and peptide mass fingerprinting analysis.
\end{abstract}

Correspondence to: Professor Gon Sup Kim, Research Institute of Life Science and College of Veterinary Medicine (BK21 Plus Project), Gyeongsang National University, 900 Gajwa-dong, Jinju, Gyeongnam 660-701, Republic of Korea

E-mail: gonskim@gnu.ac.kr

Professor Won Sup Lee, Department of Internal Medicine, Institute of Health Sciences and Gyeongnam Regional Cancer Center, Gyeongsang National University School of Medicine, 90 Chilam-dong, Jinju, Gyeongnam 660-702, Republic of Korea

E-mail: lwshmo@hanmail.net; lwshmo@gnu.ac.kr

Key words: Citrus platymamma, flavonoids, lung cancer, A549 cells, proteome analysis, anticancer effects
Specifically, proteins involved in signal transduction were significantly downregulated, including annexin A1 (ANXA1) and ANXA4, whereas 14-3-3e was upregulated. Cytoskeletal proteins, including cofilin-1 (CFL1), cytokeratin 8 (KRT8) and KRT79, and molecular chaperones/heat shock proteins, including endoplasmin, were downregulated. Proteins involved in protein metabolism, namely elongation factor Ts were also downregulated. Consistent with results of the proteome analysis, the immunoblotting results showed that 14-3-3e was upregulated, whereas CFL1, ANXA4 and KRT8 were downregulated in the FCP-treated A549 cells. The majority of the proteins were involved in tumor growth, cell cycle, apoptosis, migration and signal transduction. These findings provide novel insights into the molecular mechanisms underlying FCP-induced anticancer effects on A549 cells.

\section{Introduction}

Lung cancer remains a leading cause of cancer-associated mortality worldwide, and non-small cell lung cancer (NSCLC) is a predominant type, accounting for $75-80 \%$ of lung cancer cases $(1,2)$. Although surgical resection, chemotherapy and radiotherapy are available, the long-term survival rates of patients with NSCLC remain poor. Therefore, a shift in paradigm is required from increasing the survival rates of patients with lung cancer patients to preventing lung cancer development. Previous investigations on cancer have focused on natural herbs in preventing or controlling cancer as an alternate therapy (3-5). In addition, natural substances and plant derivatives have been used to treat cancer patients with reduced toxicity. Previous reports have also suggested that flavonoids in fruit can enhance anticancer effects (6-10), indicating that these substances are suitable for chemoprevention. Citrus platymamma Hort. ex Tanaka (Byungkyul in Korean) has been used in Korean folk medicine for the treatment of various diseases, including cancer. Flavonoids, which has been reported in citrus species, including C. platymamma, exert 
antiproliferative, anticancer, antioxidant, anti-inflammatory and antidiabetic activities (11-14). However, the molecular mechanism underlying the anticancer effect of flavonoids from C. platymamma (FCP) on lung cancer remains to be elucidated.

Proteomics is now an important area of investigation in various fields, including cancer biology. Proteome analysis has been applied in the investigation of various types of cancer in-vitro and in vivo (15-19), including lung cancer (20-22). Previously, the anticancer mechanisms of therapeutic agents have been elucidated by performing comparative proteome analysis on A549 cells (23-25). The present study was conducted to investigate the mechanism of the anti-cancer effect of FCP-treated A549 cells by examining the expression of proteins involved in cancer cell survival, apoptosis, differentiation, invasion, metastasis and metabolism. Increased understanding of the molecular mechanisms underlying the anticancer effects of FCP may provide novel insights in the prevention of lung carcinogenesis, which may assist in developing novel strategies not only to prevent cancer development, but also to improve quality of life for patients with lung cancer.

Our previous study demonstrated that FCP induced G2/M cell cycle arrest and apoptosis in A549 human lung cancer cells (26). Therefore, the present study aimed to identify the differentially expressed key proteins, which may underlie the anticancer effects of FCP on A549 cells, using a proteomic approach. In total, eight differentially expressed proteins were identified using two-dimensional gel electrophoresis, coupled with matrix-assisted laser desorption/ionization time-of-flight/time-of-flight tandem mass spectrometry (MALDI-TOF/TOF-MS) analysis; 14-3-3e (YWHAE) was upregulated, and cofilin-1 (CFL1), annexin A1 (ANXA1), annexin A4 (ANXA4), endoplasmin (HSP90B1), cytoskeratin 8 (KRT8), elongation factor Ts (tsf) and uncharacterized protein (KRT79) were downregulated. The expression levels of YWHAE, CFL1, ANXA4 and KRT8 were also validated by immunoblotting. To date, this is first study, to the best of our knowledge, to use proteomic techniques to investigate the molecular mechanisms underlying the anticancer effects of FCP on A549 cells.

\section{Materials and methods}

Chemical and reagents. The A549 human lung cancer cells and WI-38 normal human fetal lung fibroblast cells were obtained from the Korea Cell Line Bank (Seoul, Korea). RPMI 1640 medium was purchased from GE Healthcare Life Sciences Hyclone Laboratories (Logan, UT, USA). Fetal bovine serum (FBS) and antibiotics (streptomycin/penicillin) were purchased from Gibco; Thermo Fisher Scientific, Inc. (Waltham, MA, USA). 5-diphenyltetrazolium bromide (MTT) was obtained from Sigma-Aldrich (St. Louis, MO, USA). Materials and chemicals used for electrophoresis were obtained from Bio-Rad Laboratories, Inc, (Hercules, CA, USA). FCP was provided by Animal Bio-Resources Bank (Jinju, Korea). Antibodies targeting CFL1 (cat. no. AB3831), ANXA4 (cat. no. ABC885) and KRT8 (cat. no. MAB1611) and $\beta$-actin (cat. no. MABT825) were purchased from EMD Millipore (Billerica, MA, USA). Antibody targeting
YWHAE (cat. no. BS6109) was obtained from Bioworld Technology, Inc. (St. Louis Park, MN, USA). Horseradish peroxidase (HRP)-conjugated goat anti-mouse $\mathrm{IgG}$ (ALX-211-205TS-C100) and anti-rabbit IgG (ADI-SAB-301-J) were purchased from Enzo Life Sciences, Inc. (Farmingdale, NY, USA). All other chemicals used in the present study were purchased from Amresco, Inc. (Solon, OH, USA) and Sigma-Aldrich. All chemicals used were of the highest grade commercially available.

Cytotoxicity assay. The A549 cells and WI-38 cells ( $\left.1 \times 10^{5}\right)$ were grown in RPMI-1640 medium supplemented with $10 \%$ heat-inactivated FBS and $1 \%$ penicillin/streptomycin in a humidified incubator with $5 \% \mathrm{CO}_{2}$ in air $37^{\circ} \mathrm{C}$. The cells were cultured in 12-well plates and incubated overnight. Subsequently, the cells were treated with 0, 100, 200, 300, 400 and $500 \mu \mathrm{g} / \mathrm{ml}$ of FCP for $24 \mathrm{~h}$. Following incubation, $100 \mathrm{ml}$ of $0.5 \mathrm{mg} / \mathrm{ml} \mathrm{MTT} \mathrm{solution} \mathrm{was} \mathrm{added} \mathrm{to} \mathrm{each} \mathrm{well}$ and incubated for $3 \mathrm{~h}$ at $37^{\circ} \mathrm{C}$ in the dark. The formazan contained in the cells was solubilized by the addition of $500 \mathrm{ml}$ of DMSO, and the absorbance was measured at $540 \mathrm{~nm}$ using an enzyme-linked immunosorbent assay plate reader.

Preparation of the total cellular extract for 2-DE. The total proteins were extracted from the A549 cells in the untreated (control) and FCP-treated groups. Following incubation with FCP, the cells were lysed in lysis buffer, containing $7 \mathrm{M}$ urea, $2 \mathrm{M}$ thiourea and $4 \%$ (w/v) CHAPS, on ice for $1 \mathrm{~h}$. The lysates were then centrifuged at $13,000 \times \mathrm{g}$ for $15 \mathrm{~min}$ at $4^{\circ} \mathrm{C}$, and the collected supernatant was stored at $-80^{\circ} \mathrm{C}$ until analysis. The total protein was used for $2-\mathrm{DE}$. The protein concentration was determined using the Non-Interfering ${ }^{\mathrm{TM}}$ protein assay kit (G-Biosciences, St. Louis, MO, USA), in accordance with the manufacturer's protocol.

2-DE and image analysis. An equal quantity $(150 \mu \mathrm{g})$ of protein per sample was loaded onto a $18 \mathrm{~cm}$ linear IPG strip (pH 4-7; Amersham Biosciences; Uppsala, Sweden) for first-dimensional isoelectrofocusing, which was followed by $12 \%$ second dimension sodium dodecyl sulfate polyacrylamide gel electrophoresis (SDS-PAGE) on an Ettan DALT II system (Amersham Biosciences). The gels were stained with silver nitrate, as described previously with modifications (27), and three independent gels were used in triplicate. Briefly, gels were incubated in fixation solution (50\% ethanol and $5 \%$ acetic acid) for $15 \mathrm{~h}$, washed once with $30 \%$ ethanol for $15 \mathrm{~min}$ followed by three times with distilled water for 5 min each. The gels were stained with silver nitrate $(0.3 \%)$ in the dark for $25 \mathrm{~min}$ at room temperature. The gels were subsequently rinsed with water three times and developed with solution containing $3 \%$ sodium carbonate, $0.02 \%$ sodium thiosulfate and $0.05 \%$ formalin. The gels were scanned and image analysis was performed using Progenesis Samespots software (Nonlinear Dynamics, Newcastle, UK). Using this software, the differentially expressed spots were identified by automatic matching of the detected protein spots. Those spots differing significantly $(\mathrm{P}<0.05)$ in their intensities (fold-change $\geq 2$ ), in the FCP-treated A549 cells were used for further analysis. 
MALDI-TOF/ TOF MS analysis. Selected protein spots were excised manually from the 2-DE gel, and protein digestion was performed (28) with modifications. Briefly, the excised gel pieces were washed with $100 \mu \mathrm{l} 100 \mathrm{mM} \mathrm{NH}_{4} \mathrm{HCO}_{3}$ for $5 \mathrm{~min}$ and then dehydrated in $100 \mu \mathrm{l}$ acetonitrile for $10 \mathrm{~min}$. Following drying in a lyophilizer (SFDSM06; Samwon Freezing Engineering Co., Busan, South Korea), the gel pieces were rehydrated in 5-10 $\mu \mathrm{l} 50 \mathrm{mM} \mathrm{NH} \mathrm{HCO}_{3}$ containing $20 \mathrm{ng} / \mu \mathrm{l}$ trypsin (Promega Corporation, Madison, WI, USA) on ice. After $45 \mathrm{~min}$, the trypsin solution was removed and

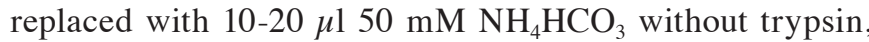
and digestion was performed for a minimum of $16 \mathrm{~h}$ at $37^{\circ} \mathrm{C}$. Subsequently, the peptide mixtures were targeted onto a MALDI-TOF/TOF plate and analyzed using a Voyager-DE STR mass spectrometer (Applied Biosystems; Thermo Fisher Scientific, Inc.), equipped with delay ion extraction.

Database search. The proteins were identified using the Mascot program (http://www.matrixscience.com). The Swissprot database and peptide mass fingerprinting (PMF) data were used to identify matching proteins. The following parameters were used for the database searches: Taxonomy, Homo sapiens (human); cleavage specificity, trypsin with one missed cleavage permitted; peptide tolerance of 100 ppm for the fragment ions; permitted modifications, fixed cysteine carbamidomethylation, variable oxidation of methionine. Protein scores $>84$ were considered statistically significant $(\mathrm{P}<0.05)$.

Western blot analysis. The A549 cells $\left(2 \times 10^{5}\right)$ were cultured in 6-well plates and incubated with FCP $(363 \mu \mathrm{g} / \mathrm{ml})$ for $24 \mathrm{~h}$. The cell lysates was prepared, and $30 \mu \mathrm{g}$ of proteins were separated by $12 \%$ SDS-PAGE and transferred onto a PVDF membrane. The blots were blocked with 5\% nonfat dry milk for $1 \mathrm{~h}$ at room temperature and then incubated with primary antibodies overnight (dilution, 1:1,000), followed by incubation with HRP-conjugated goat anti-mouse IgG (dilution, 1:1,000) for $2 \mathrm{~h}$ at room temperature. The signal was visualized using ECL detection reagent (GE Healthcare Life Sciences) and quantified by densitometry using the Image $\mathbf{J}$ (http://rsb.info. nih.gov) program. The densitometry readings of the bands were normalized to the expression of $\beta$-actin. The experiment was repeated three times.

Gene ontology (GO) analysis. GO analysis was performed using the Agbase database (http://www.agbase.msstate. edu/), as previously described (29). GO annotations were obtained from GORetriever by submitting the spot identities. The annotation results were summarized based on the GOA and whole proteome GOSlim set using GOSlimViewer (agbase.msstate.edu/cgi-bin/tools/goslimviewer_select.pl).

Statistical analysis. All statistical analyses were performed using SPSS software (SPSS for Windows, ver. 10.0; SPSS, Inc. Chicago, IL, USA). The data are presented as the mean \pm standard deviation of at least three independent experiments. The statistical significance between the control and test groups was determined using one-way analysis of variance followed by Student's $t$-test. $\mathrm{P}<0.05$ was considered to indicate a statistically significant difference.

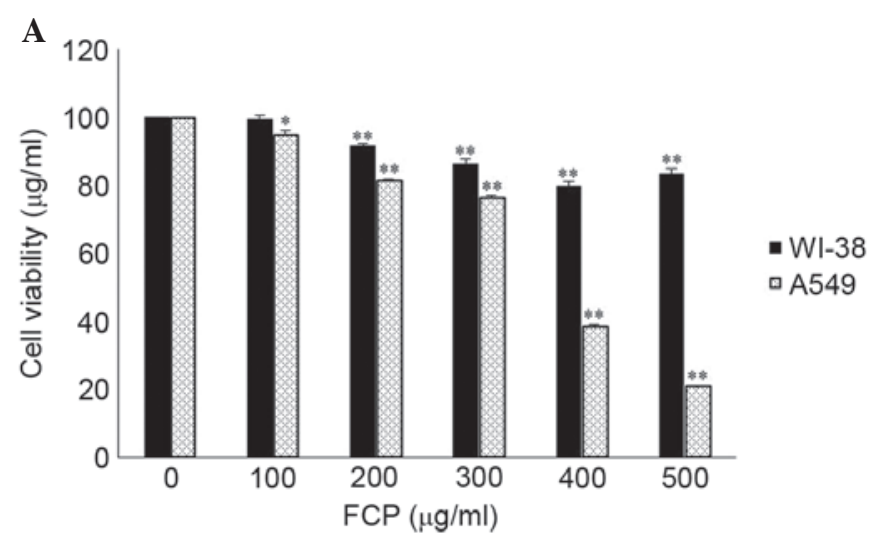

B

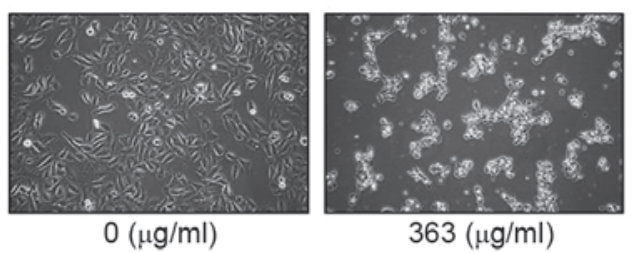

Figure 1. Cytotoxic effects of FCP against WI-38 cells and A549 human lung cancer cells. The cells were incubated at indicated concentrations of FCP for $24 \mathrm{~h}$. The cell viability was analyzed using a 5-diphenyltetrazolium bromide assay. (A) FCP showed growth inhibitory and cytotoxic effects against A549 cells in a dose-dependent manner. No cytotoxic effects were found against the WI-38 cells. (B) Morphological changes in the FCP-treated A549 cells were visualized using optical microscopy (magnification, $\mathrm{x} 400$ ). The data are shown as the mean \pm standard deviation of three independent experiments ( $\mathrm{P}<0.05$ and ${ }^{* *} \mathrm{P}<0.01$, vs. control). $\mathrm{FCP}$, flavonoids isolated from Citrus platymamma.

\section{Results}

FCP specifically inhibits the proliferation of A549 human lung cancer cells. To investigate the anticancer activity of FCP, A549 human lung cancer cells and WI-38 human fetal lung fibroblasts were treated with the indicated concentrations $(0-500 \mu \mathrm{g} / \mathrm{ml})$ of FCP for $24 \mathrm{~h}$. FCP inhibited the growth of the A549 cells in a dose-dependent manner, however, no definite cytotoxic effects were found in the WI-38 cells (Fig. 1A) and cytotoxicity was negligible at concentrations of $\geq 200 \mu \mathrm{g} / \mathrm{ml}$. These results showed that FCP exhibited a level of specific cytotoxicity towards cancer cells. The 50\% inhibitory concentration for FCP treatment was found to be $\sim 363 \mu \mathrm{g} / \mathrm{ml}$. In addition, morphological changes, including cell shrinkage and density, and decreased cell numbers were observed in the FCP-treated A549 cells (Fig. 1B). These results suggested that the FCP specifically inhibited the A549 lung cancer cells.

2-DE analysis and identification of differentially expressed proteins using MALDI-TOF/TOF-MS. Subsequently, to investigate the mechanism underlying the inhibitory effects of FCP on A549 cell proliferation, 2-DE gel analysis was performed. The representative 2-DE patterns of the untreated (control) and FCP-treated A549 cells were obtained by resolving $150 \mu \mathrm{g}$ of total proteins on IPG strips (18 cm; $\mathrm{pH} 4-7)$ in the first dimension and 12\% SDS-PAGE in the second dimension (Fig. 2). In total, 15 differentially expressed protein spots were identified $(\geq 2$-fold; $\mathrm{P}<0.05)$ using Progenesis 


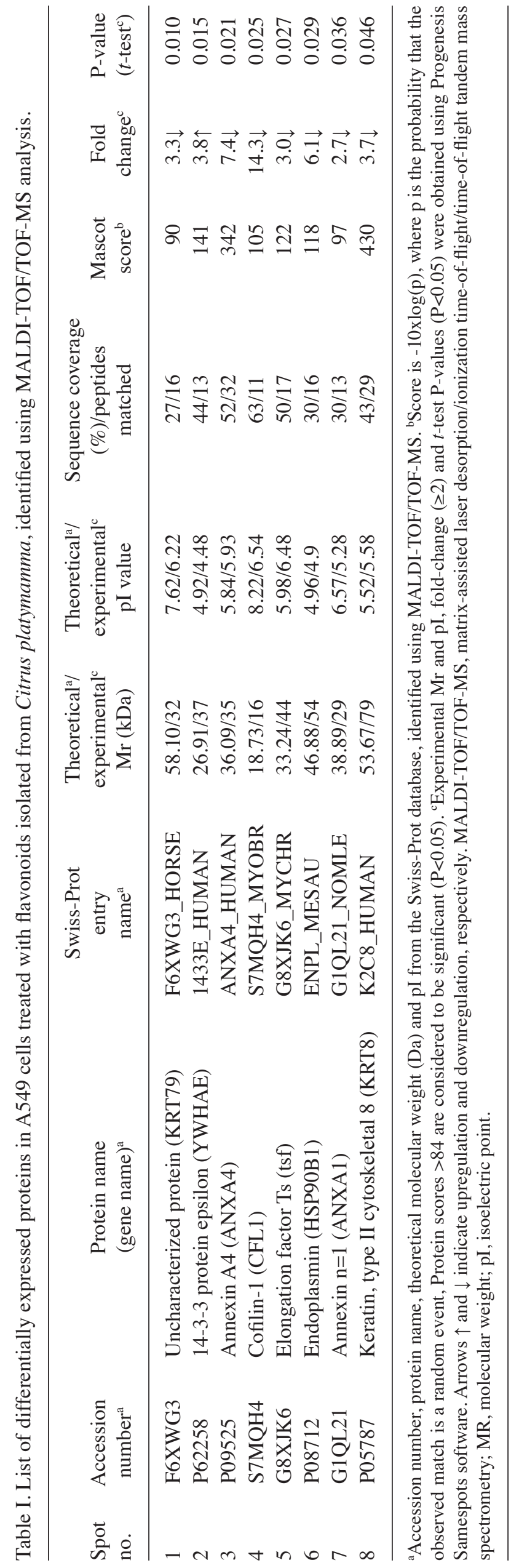


A

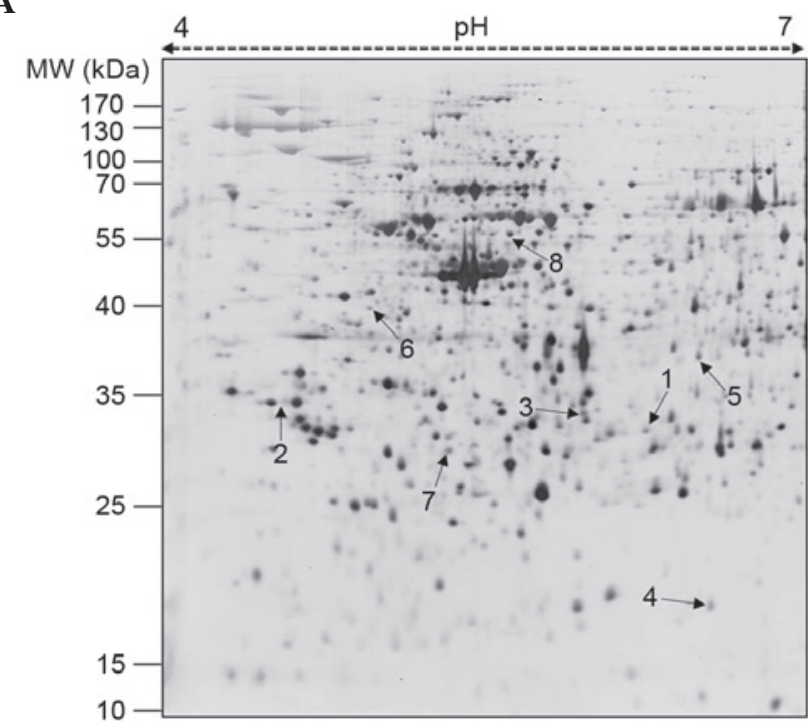

B

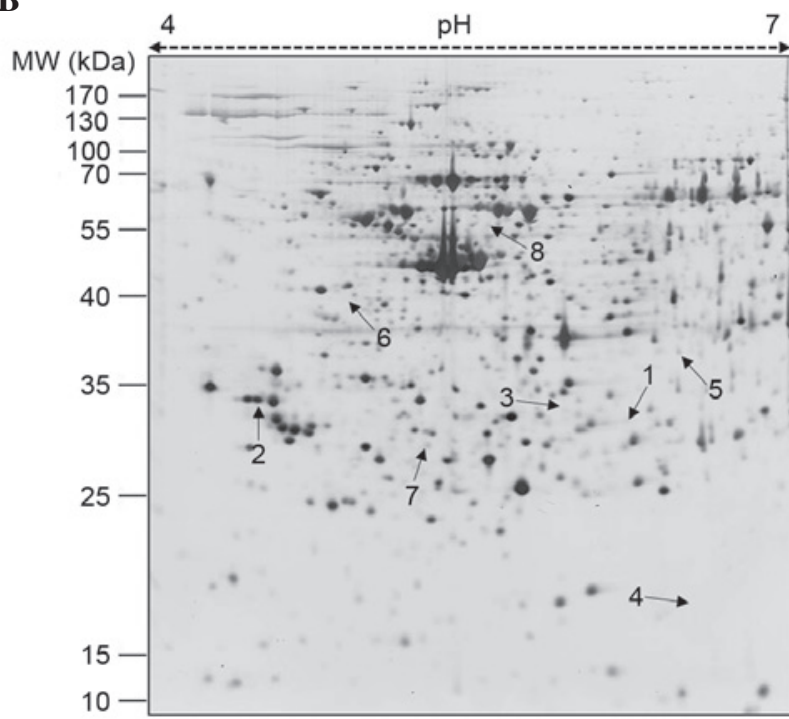

Figure 2. Two-dimensional gel electrophoresis proteome maps of the control and FCP-treated A549 cells. The (A) control and (B) FCP-treated (363 $\mu$ g/ml) A549 cells were incubated with the indicated concentrations of FCP for $24 \mathrm{~h}$. The total proteins were separated on 18 $\mathrm{cm}$ linear IPG strips (pH $4-7$ ) in the first dimension and $12 \%$ sodium dodecyl sulfate polyacrylamide gel electrophoresis in the second dimension. The gels were silver stained. The numbered arrows indicate protein spots successfully identified by matrix-assisted laser desorption/ionization time-of-flight/time-of-flight tandem mass spectrometry The experiments were performed in triplicate. FCP, flavonoids isolated from Citrus platymamma.
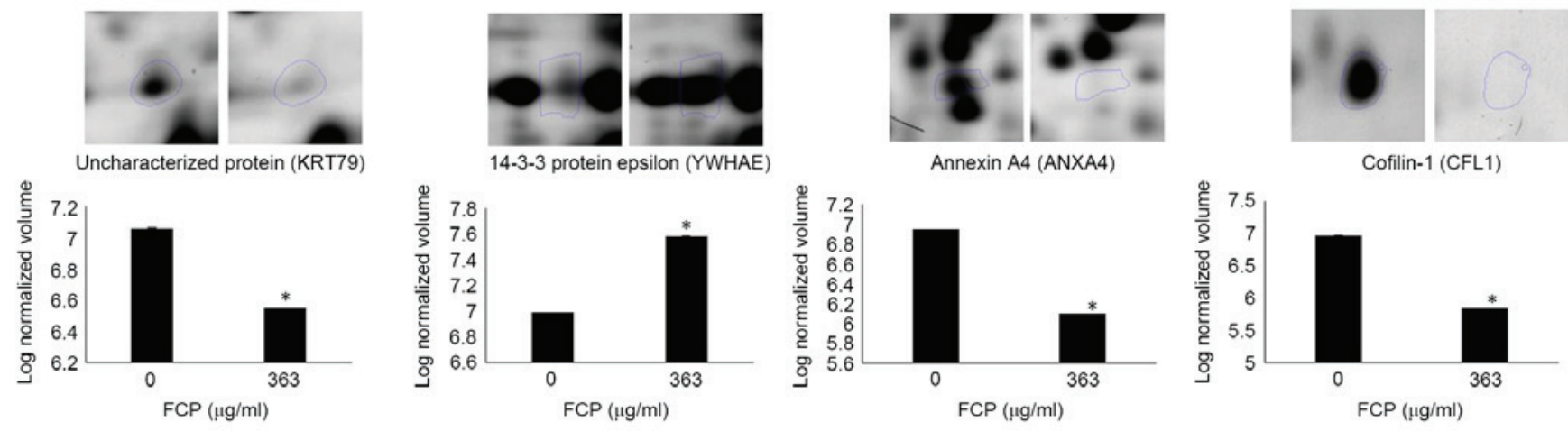

Annexin A4 (ANXA4)

Cofilin-1 (CFL1)
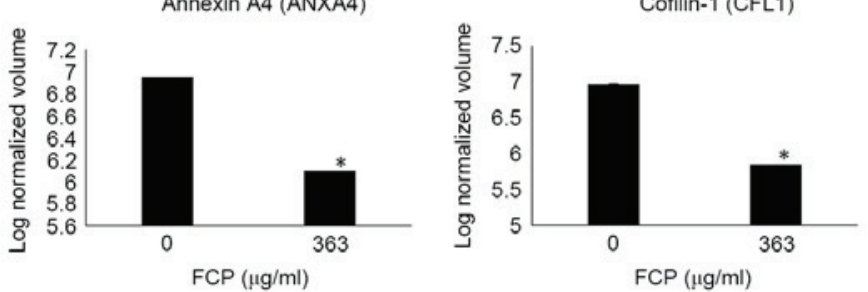

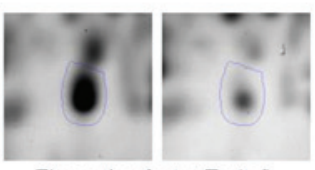

Elongation factor Ts (tsf)

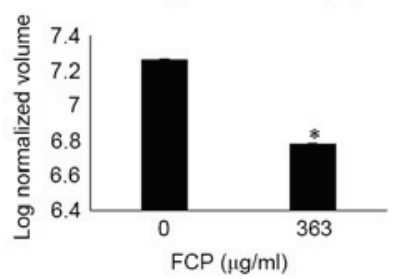

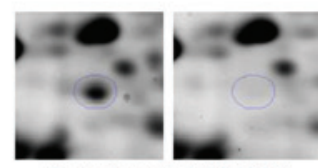

Endoplasmin (HSP90B1)

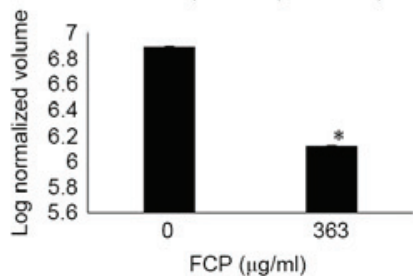

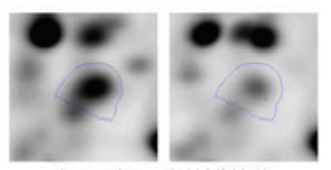

Annexin $n=1$ (ANXA1)

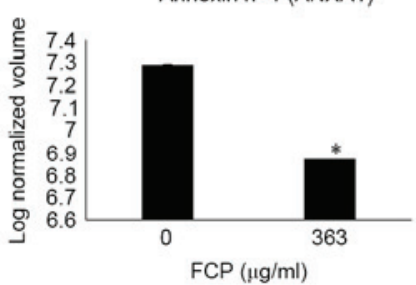

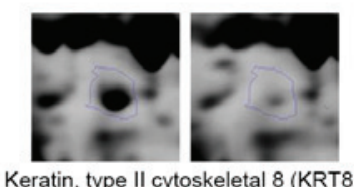

Keratin, type II cytoskeletal 8 (KRT8)

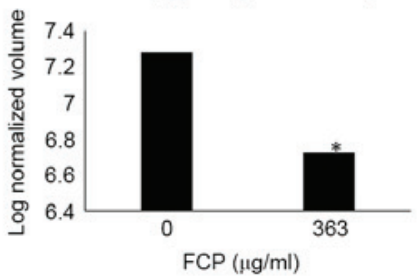

Figure 3. Expression profiles of enlarged two-dimensional gel electrophoresis spots identified in FCP-treated A549 cells. Gels were scanned and image analysis was performed using Progenesis Samespots software. The spots differing significantly in their intensities (fold-change $\geq 2$ ) in FCP-treated A549 cells, compared with untreated control cells. The data are shown as the mean \pm standard deviation of three independent experiments ( $\mathrm{P}<0.05$ vs. control). FCP, flavonoids isolated from Citrus platymamma.

Samespots image analysis software (version 4.0). Finally, eight differentially expressed proteins, one upregulated and seven downregulated, were detected in the FCP-treated A549 cells using MALDI-TOF/TOF-MS analysis and database searching (Fig. 2 and Table I). Specifically, YWHAE was upregulated, and ANXA4, CFL1, tsf, HSP90B1, ANXA1, KRT8 and KRT79 were downregulated in the FCP-treated A549 cells, compared with the control cells (enlarged 2-DE images in Fig. 3). The annotation of all identified proteins with their corresponding Swissprot accession number, experimental and theoretical 
A
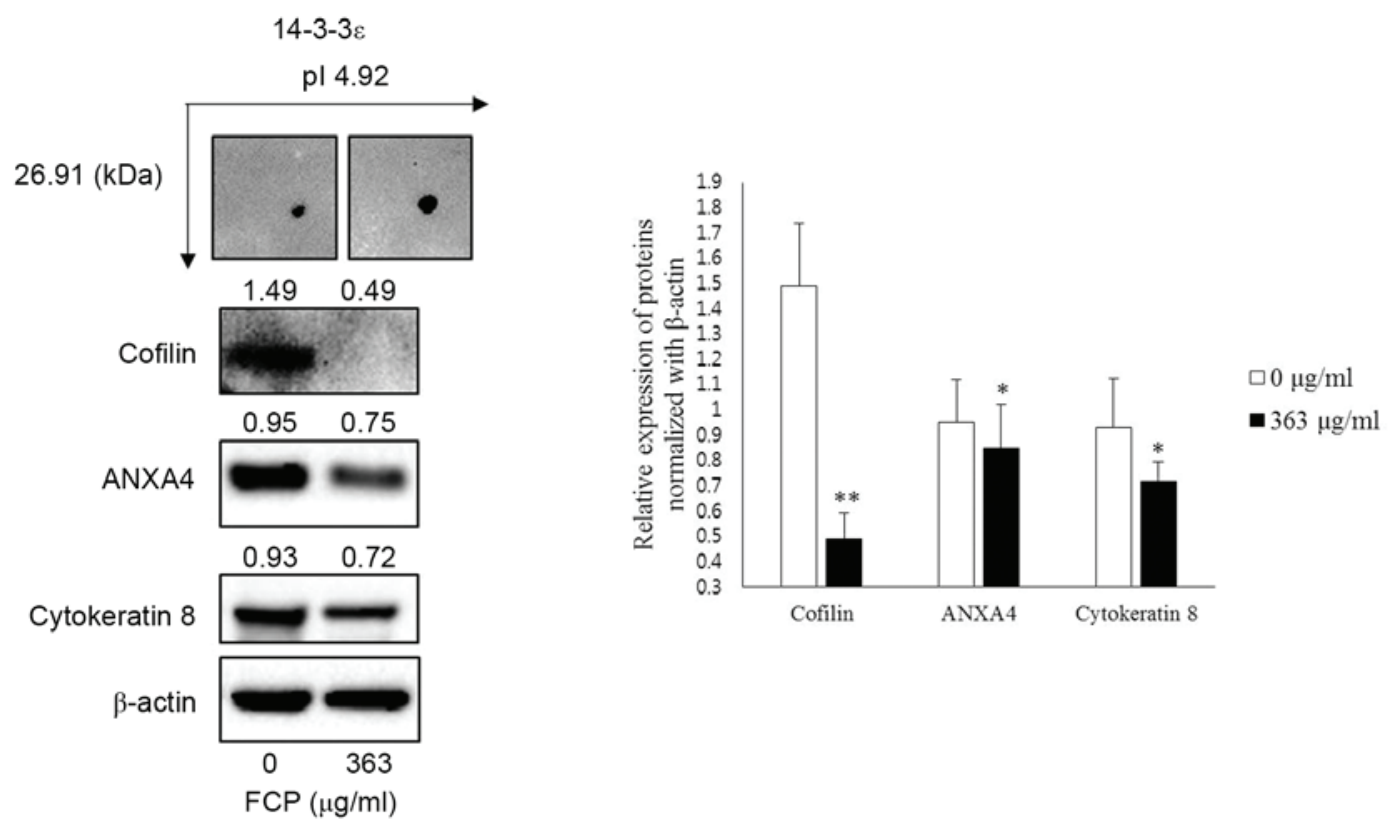

$\mathbf{B}$

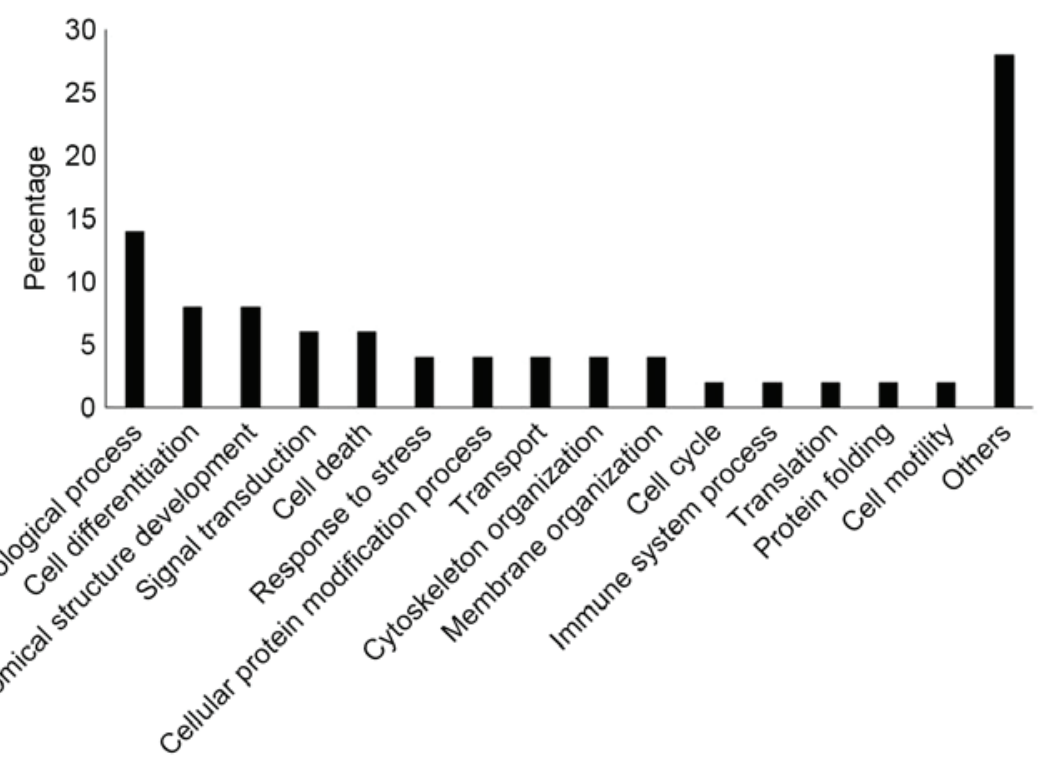

C

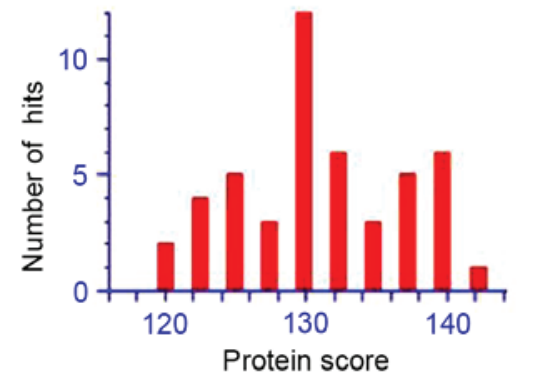

1 SMDDREDLVY QAKLAEQAER YDEMVESMKK VAGMDVELTV EERNLLSVAY

51 KNVIGARRAS WRIISSIEQK EENKGGEDKL KMIREYRQMV ETELKLICCD

101 ILDVLDKHLI PAANTGESKV FYYKMKGDYH RYLAEFATGN DRKEAAENSL

151 VAYKAASDIA MTELPPTHPI RLGLALNFSV VFYYEILNSPD RACRLAKAAF

201 DDAIAELDTL SEESYKDSTL IMQLLRDNLT LWTS

Figure 4. Validation of the results from two-dimensional gel electrophoresis using immunoblotting. The cells were incubated at indicated concentrations of FCP for 24 h. (A) Expression levels of 14-3-3e, cofilin, ANXA4 and cytokeratin 8 were assayed using western blot analysis. (B) Gene ontology analysis of the identified proteins, according to their biological function, using the GOSlimViewer tool at Agbase (http://www.agbase.msstate.edu/). (C) Top hits of protein

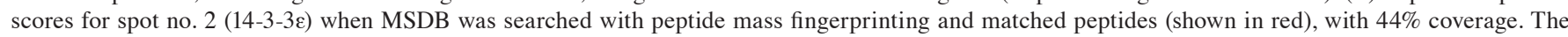
experiments were performed in triplicate $\left({ }^{*} \mathrm{P}<0.05\right.$ and ${ }^{* *} \mathrm{P}<0.01$, vs. control). FCP, flavonoids isolated from Citrus platymamma; ANXA4, annexin A4.

molecular weight, experimental and theoretical isoelectric point, sequence coverage and number of peptide matches, Mascot score, expression and statistical values are shown in
Table I. Based on protein functions, the identified proteins were divided into the following categories: Cytoskeletal proteins (CFL1, KRT8 and KRT79), signal transduction 


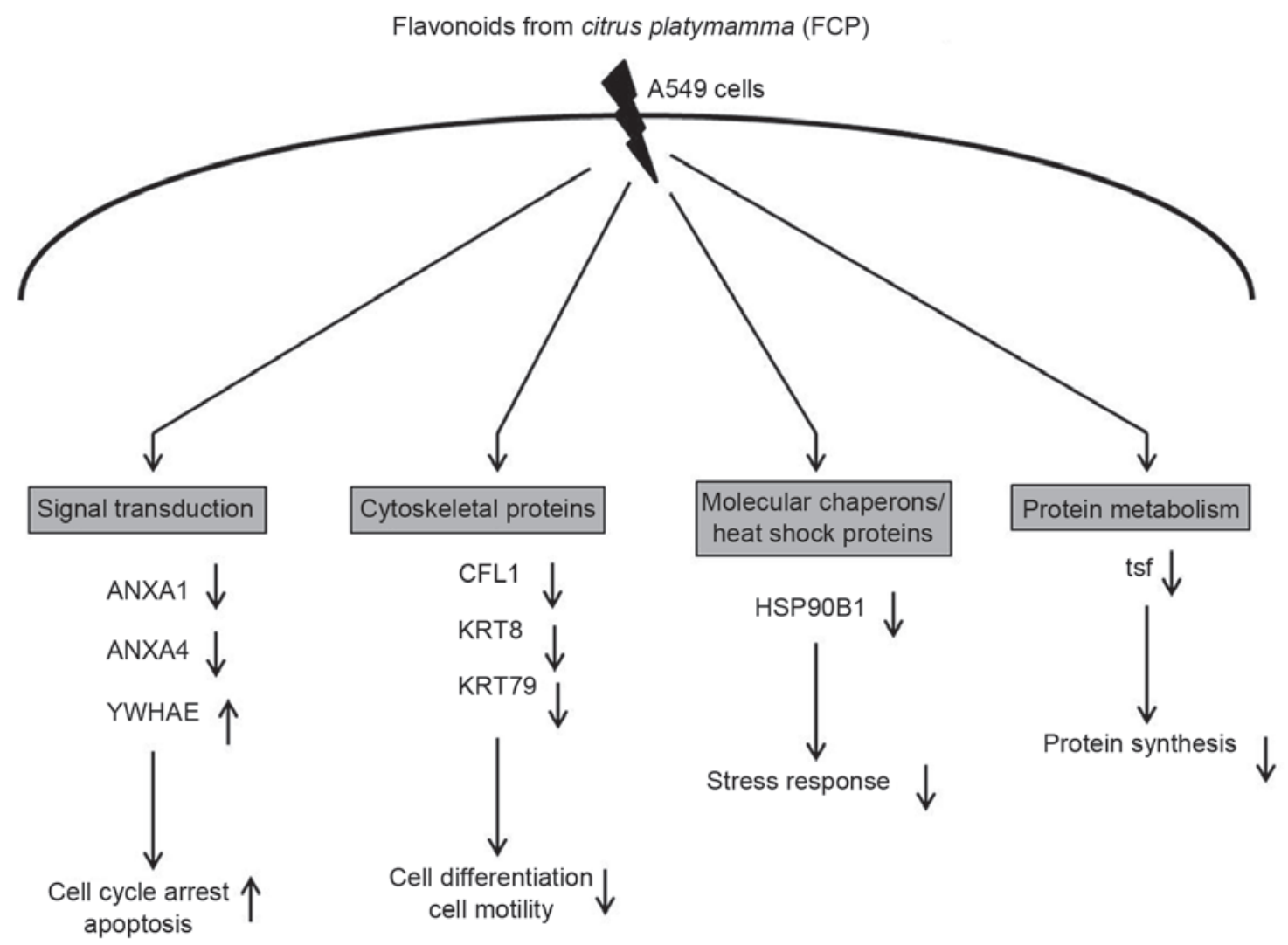

Figure 5. Biological role of eight differentially expressed proteins identified in FCP-treated A549 cells using two-dimensional gel electrophoresis coupled with matrix-assisted laser desorption/ionization time-of-flight/time-of-flight tandem mass spectrometry analysis. Specifically, proteins involved in signal transduction were significantly downregulated, including ANXA1 and ANXA4, whereas YWHAE was upregulated. Cytoskeletal proteins, including CFL1, KRT8 and KRT79, and molecular chaperones/heat shock proteins, including HSP90B1, were downregulated. Proteins involved in protein metabolism, namely tsf, were also downregulated. The majority of these proteins were involved in tumor growth and progression, cell cycle, stress response and apoptosis. ( $\uparrow$ indicates upregulation of protein and $\downarrow$ indicates downregulation of protein). ANXA, annexin A; YWHAE, 14-3-3e; CFL1, cofilin-1; KRT8, cyroskeratin 8; KRT79, uncharacterized protein; HSP90B1; endoplasmin; tsf, elongation factor Ts.

(YWHAE, ANXA1 and ANXA4), molecular chaperons/heat shock proteins (HSP90B1) and protein metabolism (tsf). The identified proteins were predominantly involved in tumor growth, cell cycle, apoptosis, migration and signal transduction.

Validation of differential expressed proteins using western blot analysis. The immunoblotting was performed to confirm the expression of proteins, which were identified in the FCP-treated A549 cells using proteome analysis. The results showed that YWHAE was significantly upregulated, whereas CFL1, ANXA4 and KRT8 were significantly downregulated in the FCP-treated A549 cells, compared with the control $(\mathrm{P}<0.05$; Fig. 4A). These findings suggested that the results of the immunoblotting were consistent with those of the comparative proteomic analysis.

GO analysis. The GO terms for biological processes were examined for all eight identified proteins. The most notable functional categories in terms of the protein expression pattern are shown in Fig. 4B. The highest associations were with biological processes (14\%; GO:0008150). Another $8 \%$ of the associations were with cell differentiation (GO:0030154) and anatomical structure development (GO:0048856), whereas 6\% were associated with signal transduction (GO:0,007165) and cell death (GO:0008219). Of the remainder, $4 \%$ were associated with response to stress (GO:0006950), cellular protein modification process (GO:0006464), cytoskeleton organization (GO:0007010), membrane organization (GO:0061024) and transport (GO:0006810).

\section{Discussion}

The present study focused on the differentially expressed proteins, which are involved in the behaviors of FCP-treated A549 cells using proteome techniques. Of 15 differentially expressed protein spots, eight proteins were successfully identified in the FCP-treated A549 cells using 2-DE coupled with MALDI-TOF/TOF-MS analyses (Fig. 2 and Table I). The identified proteins were predominantly involved in tumor growth and progression, and the apoptosis of A549 cells. These results indicated that FCP inhibited cell proliferation and induced cell death of the A549 cells by regulating those proteins. The results of the immuneoblotting confirmed that the expression of YWHAE was significantly upregulated, and the expression levels of CFL8, ANXA4 and KRT8 were significantly downregulated in the A549 cells following incubation with FCP $(363 \mu \mathrm{g} / \mathrm{ml})$ for $24 \mathrm{~h}$. These data suggested that FCP inhibited the growth of A549 cells by altering the expression of proteins, which are involved in tumor growth 
and progression. This finding is consistent with those of the previous study, demonstrating that FCP induces G2/M cell cycle arrest and apoptosis in A549 lung cancer cells.

The 14-3-3 proteins are a highly conserved protein family in eukaryotic cells, and comprise seven isoforms $(\beta, \varepsilon, \gamma, \eta, \sigma$, $\tau / \theta$ and $\zeta)$, which are crucial for regulating multiple cellular processes, including signal transduction, cell cycle regulation, apoptosis DNA repair, cytoskeletal regulation, cellular metabolism, proliferation, transcription, and redox-regulation or the stress response $(30,31)$. Among the 14-3-3 isoforms, the overexpression of YWHAE has been demonstrated in various types of human malignancy, including lung cancer $(32,33)$. In addition, the reduced expression of YWHAE in gastric cancer is associated with gastric carcinogenesis (34). In the present study, the expression of YWHAE (spot no. 2) was significantly increased in the FCP-treated A549 cells (Fig. 3). Consistent with the 2-DE results, the expression of YWHAE was further confirmed by immunoblotting analysis (Fig. 4A). Figure. 4C shows the protein scores for the top hits for YWHAE when MSDB was searched with PMF and matched peptides with $44 \%$ coverage. However, the role of the YWHAE protein in apoptosis remains controversial; another study showed that non-steroidal anti-inflammatory drugs induce apoptosis by the suppression of 14-3-3e YWHAE in colorectal cancer cells (35). Therefore, further detailed studies are needed regarding the role of the YWHAE protein in the anticancer effects of FCP on A549 cells.

The annexins, a family of phospholipid-binding proteins, involved in various physiological processes, including anticoagulation, anti-inflammatory, endocytosis and exocytosis, signal transduction, cell proliferation, differentiation and apoptosis $(36,37)$. ANXA1 is a calcium-dependent phospholipid-linked protein, differentially expressed in different types of cancer (38). The upregulation of ANXA1 in patients with lung cancer is associated with a poor clinical outcome $(39,40)$. In addition, Biaoxue et al $(41)$ demonstrated that the co-overexpression of Hsp90- $\beta$ and ANXA1 was associated with poor survival rates and lymphatic metastasis in patients with lung cancer patients. The increased expression of ANXA4 is associated with drug resistance to paclitaxel, a drug commonly used for the treatment of cancer (42). In addition, the elevated expression of ANXA4 is associated with advanced $\mathrm{T}$ stages in colorectal cancer and lymph node metastasis in human penile squamous cell carcinoma $(43,44)$. In the present study, the expression levels of ANXA1, ANXA4 and HSP90B1 (spot nos. 7, 3 and 6, respectively) were significantly downregulated in FCP-treated A549 cells (Figs. 3 and 4A). These results indicated that FCP exerted anticancer effects in A549 cells by suppressing the ANXA1, ANXA4 and HSP90B1 proteins.

In the present study, the expression of CFL1 (spot no. 4) was significantly downregulated in the FCP-treated A549 cells (Figs. 3 and 4A). CFL1, the actin regulatory protein, is important in tumor growth and progression (45). It has been reported that CFL1 is involved in tumor progression in ovarian carcinoma, almost $64 \%$ of all ovarian tumors are positive for CFL1 (46). In prostate cancer, knockdown of CFL1 was reported to increase sensitivity to docetaxel, a chemotherapeutic agent (47). In addition, the expression of KRT8 (spot no. 8) was also downregulated in FCP-treated A549 cells (Figs. 3 and 4A). The increased expression of
KRT8 was significantly associated with tumor progression, and decreased survival rates in patients with NSCLC (48). These data suggested that the downregulation of CFL1 and KRT8 may also be involved in the anticancer effect of FCP on A549 cells.

In conclusion, the present study demonstrated the anticancer effects of FCP on A549 human lung cancer cells using a proteomic approach. In the present study, eight differentially expressed proteins (YWHAE was upregulated; CFL1, ANXA1, ANXA4, HSP90B1, KRT8, Tsf and KRT79 were downregulated) were identified in the FCP-treated A549 cells, which were found to be involved in tumor growth, cell cycle, apoptosis, migration and signal transduction (Fig. 5). Furthermore, the expression levels of YWHAE, CLF1, ANXA4 and KTR8 were validated by immunoblotting. To the best of our knowledge, the present study was the first to use the proteomic technique to investigate the molecular mechanism in FCP- treated A549 cells. The findings of the present study improve understanding of the molecular mechanism underlying the selective growth inhibition of FCP on A549 cells, which may offer a therapeutic potential for the treatment of lung cancer.

\section{Acknowledgements}

This study was supported by a grant from the National Research Foundation of Korea funded by the Ministry of Science, ICT \& Future Planning (grant nos. 2012M3A9B8019303 and 2012R1A2A2A06045015) and the National R\&D Program for Cancer Control, Ministry for Health, Welfare and Family Affairs, Republic of Korea (grant no. 0820050).

\section{References}

1. Meoni G, Cecere FL, Lucherini E and Di Costanzo F: Medical treatment of advanced non-small cell lung cancer in elderly patients: A review of the role of chemotherapy and targeted agents. J Geriatr Oncol 4: 282-290, 2013.

2. Jung KW, Won YJ, Kong HJ, Oh CM, Seo HG and Lee JS: Prediction of cancer incidence and mortality in Korea, 2013. Cancer Res Treat 45: 15-21, 2013.

3. Jeong JW, Lee WS, Go SI, Nagappan A, Baek JY, Lee JD, Lee SJ, Park C, Kim GY, Kim HJ, et al: Pachymic acid induces apoptosis of EJ bladder cancer cells by DR5 up-regulation, ROS generation, modulation of Bcl-2 and IAP family members. Phytother Res 29: 1516-1524, 2015

4. Park KI, Park HS, Nagappan A, Hong GE, Lee do H, Kang SR, Kim JA, Zhang J, Kim EH, Lee WS, et al: Induction of the cell cycle arrest and apoptosis by flavonoids isolated from Korean Citrus aurantium L. in non-small-cell lung cancer cells. Food Chem 135: 2728-2735, 2012.

5. Hong GE, Kim JA, Nagappan A, Yumnam S, Lee HJ, Kim EH, Lee WS, Shin SC, Park HS and Kim GS: Flavonoids identified from Korean Scutellaria baicalensis Georgi inhibit inflammatory signaling by suppressing activation of NF- $\kappa \mathrm{B}$ and MAPK in RAW 264.7 cells. Evid Based Complement Alternat Med 2013: 912031, 2013

6. Hatcher H, Planalp R, Cho J, Torti FM and Torti SV: Curcumin: From ancient medicine to current clinical trials. Cell Mol Life Sci 65: 1631-1652, 2008.

7. Lee DH, Park KI, Park HS, Kang SR, Nagappan A, Kim JA, Kim EH, Lee WS, Hah YS, Chung HJ, et al: Flavonoids isolated from Korea Citrus aurantium L. induce G2/M phase arrest and apoptosis in human gastric cancer AGS cells. Evid Based Complement Alternat Med 2012: 515901, 2012.

8. Yumnam S, Park HS, Kim MK, Nagappan A, Hong GE, Lee HJ, Lee WS, Kim EH, Cho JH, Shin SC and Kim GS: Hesperidin induces paraptosis like cell death in hepatoblastoma, HepG2 Cells: involvement of ERK1/2 MAPK [corrected]. PLoS One 9: e101321, 2014. 
9. Han MH, Lee WS, Lu JN, Lee WS, Lu JN, Kim G, Jung JM, Ryu CH, Kim GY, Hwang HJ, Kwon TK and Choi YH: Citrus aurantium L. exhibits apoptotic effects on U937 human leukemia cells partly through inhibition of Akt. Int J Oncol 40: 2090-2096, 2012.

10. Lee HJ, Nagappan A, Park HS, Hong GE, Yumnam S, Raha S, Saralamma VV,Lee WS, KimEH and Kim GS:Flavonoids isolated from Citrus platymamma induce mitochondrial-dependent apoptosis in AGS cells by modulation of the PI3K/AKT and MAPK pathways. Oncol Rep 34: 1517-1525, 2015.

11. Nogata Y, Sakamoto K, Shiratsuchi H, Ishii T, Yano M and Ohta H: Flavonoid composition of fruit tissues of citrus species. Biosci Biotechnol Biochem 70: 178-192, 2006.

12. Benavente-García $\mathrm{O}$ and Castillo J: Update on uses and properties of citrus flavonoids: New findings in anticancer cardiovascular and anti-inflammatory activity. J Agric Food Chem 56: 6185-6205, 2008

13. Luo G, Guan X and Zhou L: Apoptotic effect of citrus fruit extract nobiletin on lung cancer cell line A549 in vitro and in vivo. Cancer Biol Ther 7: 966-973, 2008

14. Lai CS, Li S, Miyauchi Y, Suzawa M, Ho CT and Pan MH: Potent anti-cancer effects of citrus peel flavonoids in human prostate xenograft tumors. Food Funct 4: 944-949, 2013.

15. Yuan Y, Li W, Li L, Yang X, Gu R, Liu H, Huang K and Yu Y: Effects of tetrazanbigen on the protein expression in human hepatocellular carcinoma cell line QGY-7701. J Huazhong Univ Sci Technolog Med Sci 29: 304-308, 2009.

16. Liu CI, Chen CC, Chen JC, Su JH, Huang HH, Chen JY and $\mathrm{Wu}$ YJ: Proteomic analysis of anti-tumor effects of 11-dehydrosinulariolide on CAL-27 cells. Mar Drugs 9: 1254-1272, 2011.

17. Cheng YL, Zhang GY, Li C and Lin J: Screening for novel protein targets of indomethacin in HCT116 human colon cancer cells using proteomics. Oncol Lett 6: 1222-1228, 2013.

18. Li X, Wang Z, Liu J, Tang C, Duan C and Li C: Proteomic analysis of differentially expressed proteins in normal human thyroid cells transfected with PPFP. Endocr Relat Cancer 19: 681-694, 2012

19. Nagappan A, Park HS, Park KI, Hong GE, Yumnam S, Lee HJ, Kim MK, Kim EH, Lee WS, Lee WJ, et al: Helicobacter pylori infection combined with DENA revealed altered expression of p53 and 14-3-3 isoforms in Gulo-/-mice. Chem Biol Interact 206 $143-152,2013$

20. Chen ZJ, Wang SY, Chen JA and Peng XX: The influence of temperature on the protein expression of human lung cancer cell line A549. Shi Yan Sheng Wu Xue Bao 35: 179-183, 2002 (In Chinese).

21. Zhan XQ, Guan YJ, Li C, Chen ZC, Xie JY, Chen P and Liang SP: Differential proteomic analysis of human lung adenocarcinoma cell line A-549 and of normal cell line HBE. Sheng Wu Hua Xue Yu Sheng Wu Wu Li Xue Bao (Shanghai) 34: 50-56, 2002 (In Chinese).

22. Li MY, Xiao ZQ, Li C, Wu XY, Feng XP, Yi H, Li JL, Chen ZC, Chen P and Liang SP: Establishment of protein profile of human small cell lung cancer cell line NCI-H446. Ai Zheng 23 1116-1121, 2004 (In Chinese).

23. Wu H, Pan CL, Yao YC, Chang SS, Li SL and Wu TF: Proteomic analysis of the effect of Antrodia camphorata extract on human lung cancer A549 cell. Proteomics 6: 826-835, 2006.

24. Wu Q, Xu W, Cao L, Li X, He T, Wu Z and Li W: SAHA treatment reveals the link between histone lysine acetylation and proteome in nonsmall cell lung cancer A549 cells. J Proteome Res 12: 4064-4073, 2013.

25. Lu Z, Song Q, Yang J, Zhao X, Zhang X, Yang P and Kang J: Comparative proteomic analysis of anti-cancer mechanism by periplocin treatment in lung cancer cells. Cell Physiol Biochem 33: 859-868, 2014.

26. Nagappan A, Lee HJ, Saralamma VV, Park HS, Hong GE, Yumnam S, Raha S, Charles SH, Shin SC, Kim EH, et al Flavonoids isolated from Citrus platymamma induced G2/M cell cycle arrest and apoptosis of A549 human lung cancer cells. Oncol Lett (In press).

27. Swain M and Ross NW: A silver stain protocol for proteins yielding high resolution and transparent background in sodium dodecyl sulfate-polyacrylamide gels. Electrophoresis 16: 948-951, 1995
28. Shevchenko A, Wilm M, Vorm O and Mann M: Mass spectrometric sequencing of proteins silver-stained polyacrylamide gels. Anal Chem 68: 850-858, 1996.

29. McCarthy FM, Wang N, Magee GB, Nanduri B, Lawrence ML, Camon EB, Barrell DG, Hill DP, Dolan ME, Williams WP, et al: AgBase: A functional genomics resource for agriculture. BMC Genomics 7: 229, 2006.

30. Aitken A: Post-translational modification of 14-3-3 isoforms and regulation of cellular function. Semin Cell Dev Biol 22: 673-680, 2011.

31. Yang X, Lee WH, Sobott F, Papagrigoriou E, Robinson CV, Grossmann JG, Sundström M, Doyle DA and Elkins JM: Structural basis for protein-protein interactions in the 14-3-3 protein family. Proc Natl Acad Sci USA 103: 17237-17242, 2006.

32. Liu TA, Jan YJ, Ko BS, Liang SM, Chen SC, Wang J, Hsu C, Wu YM and Liou JY: 14-3-3e overexpression contributes to epithelial-mesenchymal transition of hepatocellular carcinoma. PLoS One 8: e57968, 2013

33. Qi W, Liu X, Qiao D and Martinez JD: Isoform-specific expression of 14-3-3 proteins in human lung cancer tissues. Int J Cancer 113: 359-363, 2005.

34. Leal MF, Calcagno DQ, Demachki S, Assumpção PP, Chammas R, Burbano RR and Smith Mde A: Clinical implication of 14-3-3 epsilon expression in gastric cancer. World J Gastroenterol 18: 1531-1537, 2012.

35. Liou JY, Ghelani D, Yeh S and Wu KK: Nonsteroidal anti-inflammatory drugs induce colorectal cancer cell apoptosis by suppressing 14-3-3epsilon. Cancer Res 67: 3185-3191, 2007.

36. Raynal P and Pollard HB: Annexins: The problem of assessing the biological role for a gene family of multifunctional calciumand phospholipid-binding proteins. Biochim Biophys Acta 1197: 63-93, 1994.

37. Zhang X, Liu S, Guo C, Zong J and Sun MZ: The association of annexin A2 and cancers. Clin Transl Oncol 14: 634-640, 2012.

38. Lim LH and Pervaiz S: Annexin 1: The new face of an old molecule. FASEB J 21: 968-975, 2007.

39. Rong B, Zhao C, Liu H, Ming Z, Cai X, Gao W and Yang S: Elevated serum annexin A1 as potential diagnostic marker for lung cancer: A retrospective case-control study. Am J Trans Res 6: 558-569, 2014.

40. Biaoxue R, Xiguang C and Shuanying Y: Annexin A1 in malignant tumors: Current opinions and controversies. Int J Biol Markers 29: e8-20, 2014.

41. Biaoxue R, Shuanying Y, Wei L, Zongjuan M, Xiguang C and Qiuhong Z: Co-overexpression of Hsp90- $\beta$ and annexin A1 with a significantly positive correlation contributes to the diagnosis of lung cancer. Expert Rev Mol Diagn 14: 1067-1079, 2014.

42. Han EK, Tahir SK, Cherian SP, Collins N and Ng SC: Modulation of paclitaxel resistance by annexin IV in human cancer cell lines. Br J Cancer 83: 83-88, 2000.

43. Duncan R, Carpenter B, Main LC, Telfer C and Murray GI: Characterisation and protein expression profiling of annexins in colorectal cancer. Br J Cancer 98: 426-433, 2008.

44. Zimmermann U, Balabanov S, Giebel J, Teller S, Junker H, Schmoll D, Protzel C, Scharf C, Kleist B and Walther R: Increased expression and altered location of annexin IV in renal clear cell carcinoma: A possible role in tumour dissemination. Cancer Lett 209: 111-118, 2004.

45. Kapoor S: Cofilin-1 overexpression and its role in tumor growth and progression in systemic malignancies. Int J Radiat Biol 90: 113,2014

46. Zhou J, Wang Y, Fei J and Zhang W: Expression of cofilin 1 is positively correlated with the differentiation of human epithelial ovarian cancer. Oncol Lett 4: 1187-1190, 2012.

47. Pérez-Martínez FC, Carrión B, Lucío MI, Rubio N, Herrero MA, Vázquez E and Ceña V: Enhanced docetaxel-mediated cytotoxicity in human prostate cancer cells through knockdown of cofilin-1 by carbon nanohorn delivered siRNA. Biomaterials 33: 8152-8159, 2012.

48. Fukunaga Y, Bandoh S, Fujita J, Yang Y, Ueda Y, Hojo S, Dohmoto K, Tojo Y, Takahara J and Ishida T: Expression of cytokeratin 8 in lung cancer cell lines and measurement of serum cytokeratin 8 in lung cancer patients. Lung Cancer 38: 31-38, 2002. 